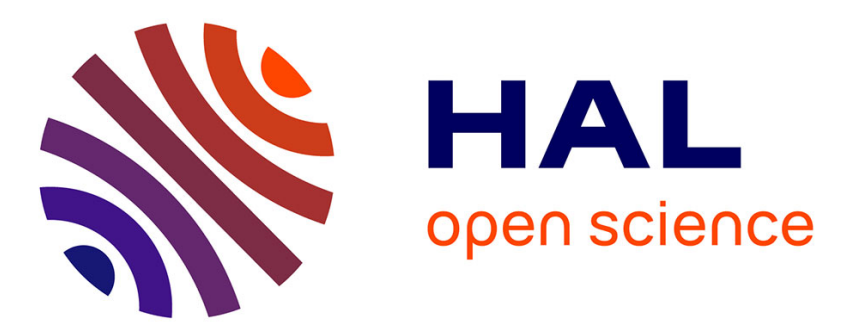

\title{
A fast precipitation and dissolution reaction for a reaction diffusion system arising in a porous medium
}

Nicolas Bouillard, Robert Eymard, Marie Henry, Raphaele Herbin, Danielle Hilhorst

\section{- To cite this version:}

Nicolas Bouillard, Robert Eymard, Marie Henry, Raphaele Herbin, Danielle Hilhorst. A fast precipitation and dissolution reaction for a reaction diffusion system arising in a porous medium. ESAIM: Mathematical Modelling and Numerical Analysis, 2007, 41 (6), pp.975-1000. 10.1051/m2an:2007047 . hal-00136237

\section{HAL Id: hal-00136237 https://hal.science/hal-00136237}

Submitted on 13 Mar 2007

HAL is a multi-disciplinary open access archive for the deposit and dissemination of scientific research documents, whether they are published or not. The documents may come from teaching and research institutions in France or abroad, or from public or private research centers.
L'archive ouverte pluridisciplinaire HAL, est destinée au dépôt et à la diffusion de documents scientifiques de niveau recherche, publiés ou non, émanant des établissements d'enseignement et de recherche français ou étrangers, des laboratoires publics ou privés. 


\title{
A fast precipitation and dissolution reaction for a reaction diffusion system arising in a porous medium *
}

\author{
N. Bouillard ${ }^{\mathrm{a}}, \mathrm{R}$. Eymard ${ }^{\mathrm{b}}$, M. Henry $^{\mathrm{c}}, \mathrm{R}$. Herbin ${ }^{\mathrm{c}}$, \\ D. Hilhorst ${ }^{\mathrm{d}}$ \\ ${ }^{a}$ CEA Saclay, DEN/DM2S/SFME, 91191 Gif sur Yvette Cedex, France \\ ${ }^{\mathrm{b}}$ Université de Marne La Vallée, 77454 Marne La Vallée cedex 2, France \\ ${ }^{\mathrm{c}}$ LATP, université de Provence, 13453 Marseille, France \\ ${ }^{\mathrm{d}}$ Department of Mathematics, université de Paris-Sud, 91405 Orsay, France
}

\begin{abstract}
This paper is devoted to the study of a fast reaction diffusion system arising in reactive transport. It extends the articles $[8,10]$ since a precipitation and dissolution reaction is considered so that the reaction term is not sign-definite and is moreover discontinuous. Energy type methods allow us to prove uniform estimates and then to study the limiting behavior of the solution as the kinetic rate tends to infinity in the special situation of one aqueous species and one solid species.
\end{abstract}

Key words: reaction-diffusion, precipitation, dissolution, kinetics, fast reaction PACS: $35 \mathrm{~K} 57$

ऋ This work was supported by GDR MOMAS

Email addresses: nicolas.bouillard@ensta.org (N. Bouillard), eymard@math.univ-mlv.fr (R. Eymard), Marie.Henry@cmi.univ-mrs.fr (M. Henry), Raphaele.Herbin@cmi.univ-mrs.fr (R. Herbin), Danielle.Hilhorst@math.u-psud.fr (D. Hilhorst). 


\section{Introduction}

In this paper we consider the reaction-diffusion system,

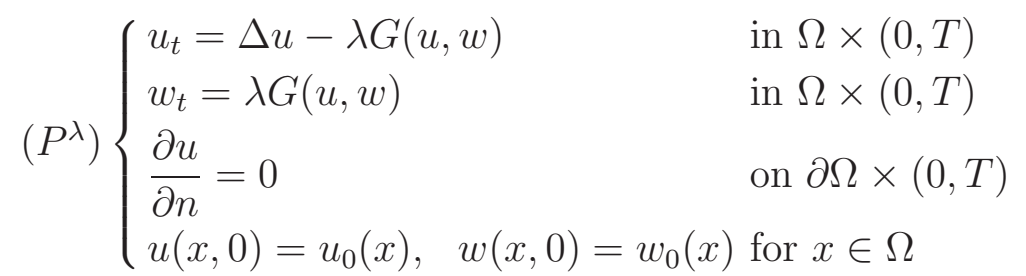

where $\Omega$ is a bounded domain in $\mathbf{R}^{N}$ with smooth boundary $\partial \Omega$ and $T$ is a positive constant. We suppose that $\lambda$ is a positive constant and that the function $G(\cdot, \cdot)$ is given by

$$
G(u, w)=(u-\bar{u})^{+}-\operatorname{sign}^{+}(w)(u-\bar{u})^{-},
$$

where $\bar{u}$ is a given positive constant and

$$
s^{+}=\max (0, s), \quad s^{-}=\max (0,-s), \text { and } \operatorname{sign}(s)= \begin{cases}1 & \text { if } s>0 \\ -1 & \text { if } s<0 \\ 0 & \text { if } s=0\end{cases}
$$

The above system $\left(P^{\lambda}\right)$ is a simplified adimensional model of reactive transport in a porous medium at the Darcy scale, where $u$ stands for a concentration of an aqueous species, therefore mobile, and $w$ stands for a concentration of a mineral species. The term $\lambda G(u, w)$ is a reaction rate that models either a precipitation if $u-\bar{u} \geq 0$, or a dissolution otherwise. The positive constant $\bar{u}$ is the thermodynamic constant of the dissolution reaction and $\lambda$ is a constant rate. Reactive transport problems arise in the field of radioactive waste storage, oil industry or $\mathrm{CO}_{2}$ storage. Indeed, water rocks interactions like precipitation and dissolution reactions have a strong impact both on flow and solute transport.

We focus on reactions which are very fast compared with the diffusion process so that $\lambda$ is a large parameter. In this paper we extend a result of Eymard, Hilhorst, van der Hout and Peletier [6], which they obtained in the special case of a function $G(\cdot, \cdot)$ assumed to be nonnegative and nondecreasing in both arguments. The Stefan problem obtained when $\lambda \rightarrow+\infty$ is the same as that of $[8,10]$ but the problem $\left(P^{\lambda}\right)$ considered in this paper has an additional precipitation term. In [8], the main tool is a finite volume method used in any space dimension. In [10], a Legendre function (associated with the liquid concentration) is used in one space dimension to deal with discontinuities. Note that in [3], the existence of a solution to the same problem with two aqueous species instead of one is proven; however, the study of the singular limit in this more complex case is still an open problem, since the techniques presented here do not seem to be easily adaptable. We suppose that the initial functions $u_{0}$ and $w_{0}$ satisfy the hypotheses: 
$\left(H_{0}\right) \quad\left\{\begin{array}{l}u_{0}, w_{0} \in L^{2}(\Omega) \quad 0 \leq u_{0} \leq M_{1} \text { and } 0 \leq w_{0} \leq M_{2} \text { a.e in } \Omega, \\ \text { for some positive constants } M_{1} \text { and } M_{2} \text { such that } M_{1}>\bar{u} .\end{array}\right.$

We set $Q_{T}:=\Omega \times(0, T)$ and denote by $W_{2}^{2,1}\left(Q_{T}\right)=\left\{u \in L^{2}\left(Q_{T}\right), \frac{\partial u}{\partial x_{i}}, \frac{\partial^{2} u}{\partial x_{i} \partial x_{j}}, \frac{\partial u}{\partial t} \in\right.$ $\left.L^{2}\left(Q_{T}\right), i, j=1, \cdots, N\right\}$ and by $C^{0,1}\left([0, T] ; L^{\infty}(\Omega)\right)$ the space of Lipschitz continuous functions with values in $L^{\infty}(\Omega)$. Next we define a notion of weak solution for Problem $\left(P^{\lambda}\right)$.

Definition $1.1\left(u^{\lambda}, w^{\lambda}\right)$ is a weak solution of Problem $\left(P^{\lambda}\right)$ if for all $T>0$

$$
\begin{aligned}
& u^{\lambda} \in W_{2}^{2,1}\left(Q_{T}\right) \quad, \quad w^{\lambda} \in C^{0,1}\left([0, T] ; L^{\infty}(\Omega)\right) ; \\
& \int_{\Omega} u^{\lambda}(T) \xi(T)-\int_{\Omega} u_{0} \xi(0)-\int_{Q_{T}}\left\{u^{\lambda} \xi_{t}-\nabla u^{\lambda} \nabla \xi-\lambda G\left(u^{\lambda}, w^{\lambda}\right) \xi\right\}=0, \\
& \int_{\Omega} w^{\lambda}(T) \xi(T)-\int_{\Omega} w_{0} \xi(0)-\int_{Q_{T}}\left\{w^{\lambda} \xi_{t}+\lambda G\left(u^{\lambda}, w^{\lambda}\right) \xi\right\}=0,
\end{aligned}
$$

for all $\xi \in H^{1}\left(Q_{T}\right)$.

The existence of a nonnegative solution pair is proven in [3] for the case of two aqueous species; the system studied in this latter case fully contains the one studied here. In view of its regularity, we remark that it satisfies the differential equations in Problem $\left(P^{\lambda}\right)$ a.e. in $Q_{T}$. The purpose of this paper is to prove the following result.

Theorem 1 Suppose that $u_{0}$ and $w_{0}$ satisfy the hypotheses $\left(H_{0}\right)$. Then for every $\lambda>0$, Problem $\left(P^{\lambda}\right)$ has a unique nonnegative weak solution $\left(u^{\lambda}, w^{\lambda}\right)$. Moreover there exist functions $U \in$ $L^{2}\left(Q_{T}\right), W \in L^{2}\left(Q_{T}\right)$ such that $u^{\lambda}$ and $w^{\lambda}$ converge strongly in $L^{2}\left(Q_{T}\right)$ to $U$ and $W$ respectively, as $\lambda$ tends to $\infty$. The function $Z:=-(U+W)+\bar{u}$ is the unique weak solution of the Stefan problem

$$
(S P)\left\{\begin{array}{lr}
Z_{t}=\Delta\left(Z^{+}\right) & \text {in } \Omega \times(0, T) \\
\frac{\partial Z^{+}}{\partial n}=0 & \text { on } \partial \Omega \times(0, T) \\
Z(x, 0)=-\left(u_{0}(x)+w_{0}(x)\right)+\bar{u} & \text { for } x \in \Omega .
\end{array}\right.
$$

Conversely the limit pair $(U, W)$ is given by $(U, W)=\left(-Z^{+}+\bar{u}, Z^{-}\right)$.

Let us first present the results of numerical simulations which show the behaviour of the solution to Problem $\left(P^{\lambda}\right)$ as $\lambda$ becomes larger, and which were obtained by solving a nonlinear system obtained from a discretization of $\left(P^{\lambda}\right)$ by the finite volume method [7], for finite values of $\lambda$. For $\lambda=\infty$, we discretized by the finite volume method the Problem $(S P)$. The physical domain $\Omega$ is the unit square $(0,1) \times(0,1)$. The initial condition is taken to be $u_{0}=0$, except in two squares where $u_{0}=2.10^{-3}, w_{0}=0$ and $\bar{u}=10^{-3}$. In these squares, since $u_{0}>\bar{u}$, then at initial time, the mineral precipitates, and therefore $w$ increases. In the sequel of the simulation, we observe the dissolution of $w$. In figures 1 and 2, we display concentration maps of $w$ after 1 time step (precipitation has occured) and 6 time steps (dissolution has occured). We select the values $\lambda=10^{3}(\mathrm{a}), 10^{6}(\mathrm{~b})$ and $\lambda=+\infty(\mathrm{c})$. When $\lambda$ increases the precipitation front gets stiffer. Indeed in Figure 2, $\max (w)$ is $6.510^{-4}(\mathrm{a}), 9.810^{-4}(\mathrm{~b}), \bar{u}=10^{-3}(\mathrm{c})$. Therefore, we observe the 
expected behaviour of the solution as $\lambda$ increases.
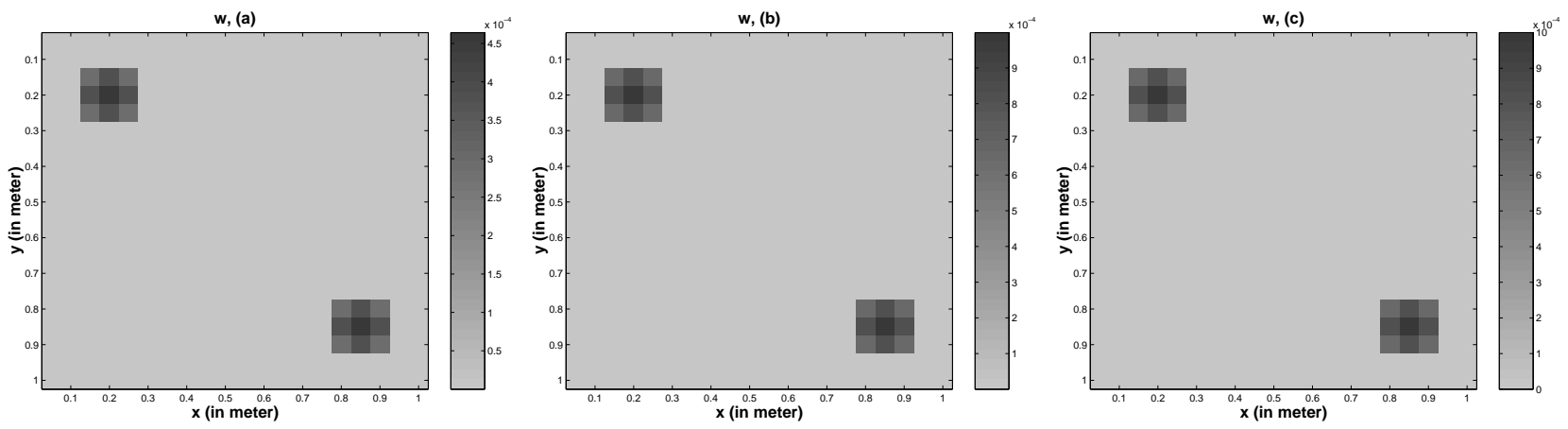

Fig. 1. Concentration of $w$ after 1 time step for $10^{3}(\mathrm{a}), 10^{6}(\mathrm{~b}), \lambda=\infty$ (c).
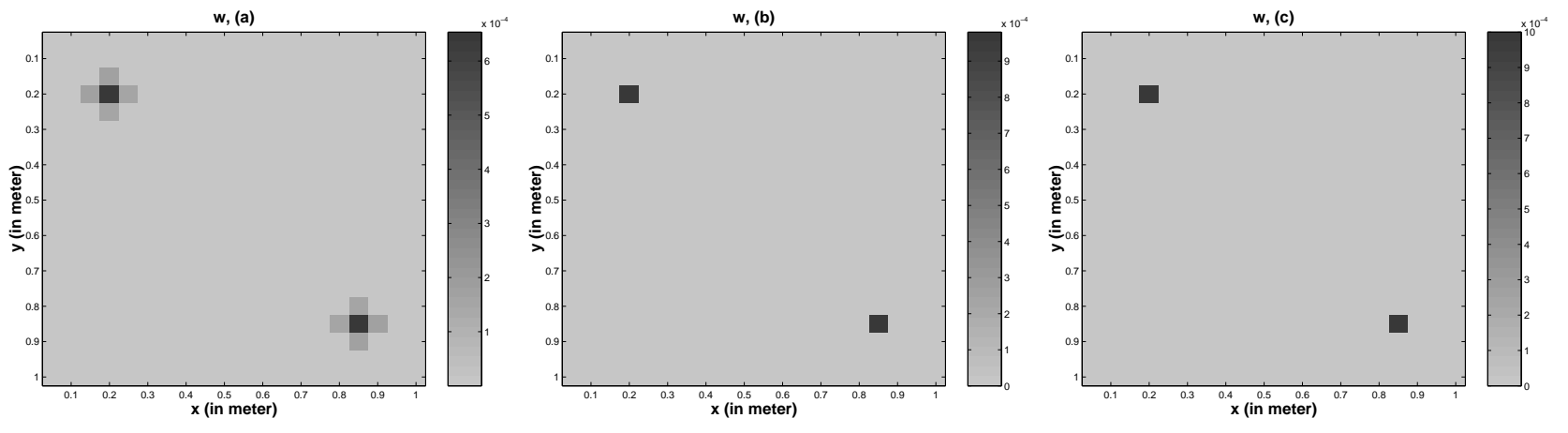

Fig. 2. Concentration of $w$ after 6 time steps for $10^{3}(\mathrm{a}), 10^{6}(\mathrm{~b}), \lambda=\infty$ (c).

The mathematical justification of this asymptotic behaviour is obtained by proving Theorem 1: this is the aim of the remainder of this article. This article is organized as follows : In Section 2 we prove a comparison principle for Problem $\left(P^{\lambda}\right)$, which implies the uniqueness of its weak solution. This result is quite natural since the monotonicity properties of $G$ in $u$ and in $w$ make it a cooperative system [1]. In Section 3 we present some a priori estimates, which imply that as $\lambda$ tends to $\infty$, the sum $-\left(u^{\lambda}+w^{\lambda}\right)+\bar{u}$ tends to the unique weak solution of the Stefan problem $(S P)$.

\section{Comparison principle and uniqueness}

We first prove the following comparison principle.

Theorem 2 Let $(u, w)$ and $(\phi, \psi)$ be such that $u, \phi \in W_{2}^{2,1}\left(Q_{T}\right)$ and $w, \psi \in C^{0,1}\left([0, T] ; L^{\infty}(\Omega)\right)$ and suppose that they satisfy 


$$
\begin{aligned}
u_{t} \geq \Delta u-\lambda G(u, w) & \text { a.e in } Q_{T} \\
w_{t} \geq \lambda G(u, w) & \text { a.e in } Q_{T} \\
\phi_{t} \leq \Delta \phi-\lambda G(\phi, \psi) & \text { a.e in } Q_{T} \\
\psi_{t} \leq \lambda G(\phi, \psi) & \text { a.e in } Q_{T} \\
\frac{\partial u}{\partial n}=\frac{\partial \phi}{\partial n}=0 & \text { a.e } \text { on } \partial \Omega \times(0, T) \\
u(x, 0) \geq u_{0}(x) \geq \phi(x, 0) & \text { for } x \in \Omega \\
w(x, 0) \geq w_{0}(x) \geq \psi(x, 0) & \text { for } x \in \Omega
\end{aligned}
$$

Then

$$
\begin{gathered}
u(x, t) \geq \phi(x, t) \quad \text { a.e in } Q_{T} \\
w(x, t) \geq \psi(x, t) \text { a.e in } Q_{T} .
\end{gathered}
$$

Before presenting the proof, we recall a technical result stated by Crandall and Pierre [5].

Lemma 2.1 Let $p: \mathbf{R} \rightarrow \mathbf{R}$ be Lebesgue measurable and bounded and define $q$ by $q(r)=$ $\int_{0}^{r} p(s) d s$. Let $\omega \in W^{1,1}\left(0, T, L^{1}(\Omega)\right)$. Then $q(\omega) \in W^{1,1}\left(0, T, L^{1}(\Omega)\right)$ and

$$
\frac{d}{d t} q(\omega)=p(\omega) \frac{d}{d t} \omega \text { a.e. }
$$

This Lemma will be used several times in this article either with $p(s)=\operatorname{sign}(s)$ and thus $q(s)=|s|$ or with $p(s)=\operatorname{sign}^{+}(s)$ and thus $q(s)=s^{+}$.

Proof. We subtract the inequality for $u$ (2.9) from that for $\phi(2.11)$, and multiply the result by $\operatorname{sign}^{\delta,+}(\phi-u)$; similarly we subtract the inequality for $\psi(2.12)$ from that for $w(2.10)$, and multiply the result by $\operatorname{sign}^{\delta,+}(\psi-w)$, where $\operatorname{sign}^{\delta,+}$ is a smooth nondecreasing regularization of sign ${ }^{+}$which converges pointwise. This approximation can be built as in Lemma 3.1 below. Adding both inequalities and integrating the result on $\Omega$ we deduce that

$$
\int_{\Omega} \frac{\partial}{\partial t} a_{\delta}(\phi-u)+\int_{\Omega} \frac{\partial}{\partial t} a_{\delta}(\psi-w) \leq \int_{\Omega} \Delta(\phi-u) \operatorname{sign}^{\delta,+}(\phi-u)+\lambda \int_{\Omega} \tau_{\delta}(u, w, \phi, \psi)
$$

with $a_{\delta}(s)=\int_{0}^{s} \operatorname{sign}^{\delta,+}(r) d r$ which converges to $s^{+}$and

$$
\tau_{\delta}(u, w, \phi, \psi)=(G(u, w)-G(\phi, \psi)) \operatorname{sign}^{\delta,+}(\phi-u)+(G(\phi, \psi)-G(u, w)) \operatorname{sign}^{\delta,+}(\psi-w) .
$$

Then,

$$
\frac{d}{d t} \int_{\Omega}\left\{a_{\delta}(\phi-u)+a_{\delta}(\psi-w)\right\} \leq-\int_{\Omega}|\nabla(\phi-u)|^{2}\left\{\operatorname{sign}^{\delta,+}(\phi-u)\right\}^{\prime}+\lambda \int_{\Omega} \tau_{\delta}(u, w, \phi, \psi) .
$$


This implies, in view of Lemma 2.2, Lebesgue's dominated convergence theorem and the hypotheses on the initial data, that for all $t \in[0, T]$,

$$
\iint_{Q_{T}}(\phi-u)^{+}(x, t) d x+\int_{\Omega}(\psi-w)^{+}(x, t) d x \leq 0
$$

so that $\phi \leq u$ and $\psi \leq w$ on $Q_{T}$.

Lemma 2.2 Let the function $\tau_{\delta}$ be defined by

$$
\tau_{\delta}(u, w, \phi, \psi)=(G(u, w)-G(\phi, \psi)) \operatorname{sign}^{\delta,+}(\phi-u)+(G(\phi, \psi)-G(u, w)) \operatorname{sign}^{\delta,+}(\psi-w),
$$

with $\operatorname{sign}^{\delta,+}$ a smooth approximation of sign ${ }^{+}$. Then $\lim _{\delta \downarrow 0} \tau_{\delta} \leq 0$.

Proof. We use the monotonicity of $G(u, w)(\nearrow u, \searrow w)$. We rewrite $\tau_{\delta}$ in the form,

$$
\begin{aligned}
\tau_{\delta}= & \{G(u, w)-G(\phi, w)\} \operatorname{sign}^{\delta,+}(\phi-u)+\{G(\phi, w)-G(\phi, \psi)\} \operatorname{sign}^{\delta,+}(\phi-u) \\
& +\{G(\phi, \psi)-G(\phi, w)\} \operatorname{sign}^{\delta,+}(\psi-w)+\{G(\phi, w)-G(u, w)\} \operatorname{sign}^{\delta,+}(\psi-w) \\
=\quad & \left.-\{G(\phi, w)-G(u, w))\} \operatorname{sign}^{\delta,+}(\phi-u)+\{G(\phi, w)-G(u, w))\right\} \operatorname{sign}^{\delta,+}(\psi-w) \\
& -\{G(\phi, w)-G(\phi, \psi)\} \operatorname{sign}^{\delta,+}(\psi-w)+\{G(\phi, w)-G(\phi, \psi)\} \operatorname{sign}^{\delta,+}(\phi-u)
\end{aligned}
$$

Applying Lebesgue's dominated convergence theorem we deduce that

$$
\int_{\Omega} \tau_{\delta}(u, w, \phi, \psi) \rightarrow \int_{\Omega} \tau(u, w, \phi, \psi) \text { as } \delta \downarrow 0,
$$

where

$$
\begin{aligned}
\tau= & -\{G(\phi, w)-G(u, w)\}^{+}+\{G(\phi, w)-G(u, w)\} \operatorname{sign}^{+}(\psi-w) \\
& -\{G(\phi, w)-G(\phi, \psi)\}^{+}+\{G(\phi, w)-G(\phi, \psi)\} \operatorname{sign}^{+}(\phi-u) \leq 0 .
\end{aligned}
$$

Corollary 2.3 Under hypotheses $\left(H_{0}\right)$, let $\left(u^{\lambda}, w^{\lambda}\right)$ be a weak solution of Problem $\left(P^{\lambda}\right)$, then:

$$
\begin{aligned}
u^{\lambda}(x, t) & \leq \tilde{u}(t):=\bar{u}+\left(M_{1}-\bar{u}\right) \mathrm{e}^{\lambda t} \text {, a.e in } Q_{T} \\
w^{\lambda}(x, t) & \leq \tilde{w}(t):=M_{2}+\left(M_{1}-\bar{u}\right)\left(1-\mathrm{e}^{\lambda t}\right) \text {, a.e in } Q_{T} .
\end{aligned}
$$

Proof. We check that $(\tilde{u}, \tilde{w})$ is the weak solution of Problem $\left(P^{\lambda}\right)$ with the constant initial data $(\tilde{u}(x, 0), \tilde{w}(x, 0))=\left(M_{1}, M_{2}\right)$. The result then follows from the comparison principle given in Theorem 2. 


\section{A priori estimates}

The purpose of this section is to prove the convergence Theorem 1. We first introduce some notations and give technical lemmas. To begin with we construct a smooth nondecreasing approximation of the sign function which we denote by $\operatorname{sign}^{\delta}$ and which converges pointwise to the function sign; we then define by $\mathcal{H}^{\delta}$ the regularization of the Heaviside function $\mathcal{H}^{\delta}(s):=$ $\int_{0}^{s} \operatorname{sign}^{\delta}(\tau) d \tau$

Lemma 3.1 There exist a sequence of smooth nondecreasing functions $\left(\operatorname{sign}^{\delta}\right)$ which converges pointwise to the function sign.

Proof. First we introduce two smooth functions $f_{0}$ and $f_{1}$ defined respectively by

$$
f_{1}(x)=\left\{\begin{array}{ll}
e^{-\frac{1}{(x-1)^{2}}} & \text { for } x<1 \\
0 & \text { for } x \geq 1
\end{array} \text { and } f_{0}(x)= \begin{cases}e^{-\frac{1}{x^{2}}} & \text { for } x \geq 0 \\
0 & \text { for } x<0\end{cases}\right.
$$

The function $f_{0} f_{1} \in C^{\infty}(\mathbf{R})$ and is equal to zero on $\mathbf{R} \backslash[0,1]$. Moreover defined $F$ by

$$
F(x)=\frac{1}{\int_{0}^{1} f_{0}(x) f_{1}(x) d x} \int_{0}^{x} f_{0}(x) f_{1}(x) d x
$$

one can check that $F \in C^{\infty}(\mathbf{R})$ and satisfies $F(0)=0, F(x)=0$ for $x<0$ and $F(x)=1$ for $x \geq 1$. Finally, setting $\operatorname{sign}^{\delta}(x)=F\left(\frac{x}{\delta}\right)$ for $\delta>0$, we deduce that $\operatorname{sign}^{\delta}(x)$ converges to $\operatorname{sign}(x)$ for all $x \in \mathbf{R}$ as $\delta$ tends to 0 .

We now prove some a priori estimates.

Lemma 3.2 Let $\left(u^{\lambda}, w^{\lambda}\right)$ be the solution of $\left(P^{\lambda}\right)$. Then there exists $C_{1}>0$ only depending on $T, \Omega$ and $\bar{u}$, such that

$$
\iint_{Q_{T}}\left|w_{t}^{\lambda}\right| d x d t=\lambda \iint_{Q_{T}}\left|G\left(u^{\lambda}, w^{\lambda}\right)\right| d x d t \leq C_{1}
$$

and

$$
\iint_{Q_{T}}\left|\nabla u^{\lambda}\right|^{2} d x d t \leq C_{1}
$$

Proof. We first prove (3.20). Multiplying (1.1) by $\operatorname{sign}^{\delta}(u-\bar{u})$ and integrating the result on $\Omega$ we obtain

$$
\frac{\mathrm{d}}{\mathrm{d} t} \int_{\Omega}\left\{\mathcal{H}^{\delta}\left(u^{\lambda}-\bar{u}\right)\right\}=-\int_{\Omega}\left\{\nabla\left(u^{\lambda}-\bar{u}\right)\right\}^{2}\left\{\operatorname{sign}^{\delta}\right\}^{\prime}\left(u^{\lambda}-\bar{u}\right)-\lambda \int_{\Omega} G\left(u^{\lambda}, w^{\lambda}\right) \operatorname{sign}^{\delta}\left(u^{\lambda}-\bar{u}\right) .
$$

Using the nonnegativity of $s \mapsto\left\{\operatorname{sign}^{\delta}\right\}^{\prime}$, we have the following inequality 


$$
\frac{\mathrm{d}}{\mathrm{d} t} \int_{\Omega}\left\{\mathcal{H}^{\delta}\left(u^{\lambda}-\bar{u}\right)\right\}+\lambda \int_{\Omega} G\left(u^{\lambda}, w^{\lambda}\right) \operatorname{sign}^{\delta}\left(u^{\lambda}-\bar{u}\right) \leq 0
$$

which we integrate on $(0, t), 0<t \leq T$ to obtain

$$
\int_{\Omega}\left\{\mathcal{H}^{\delta}\left(u^{\lambda}-\bar{u}\right)\right\}(t)+\lambda \int_{Q_{t}} G\left(u^{\lambda}, w^{\lambda}\right) \operatorname{sign}^{\delta}\left(u^{\lambda}-\bar{u}\right) \leq \int_{\Omega}\left\{\mathcal{H}^{\delta}\left(u_{0}-\bar{u}\right)\right\} .
$$

It then follows from Lebesgue's dominated convergence theorem that

$$
\int_{\Omega}\left|u^{\lambda}-\bar{u}\right|(t)+\lambda \int_{Q_{t}} G\left(u^{\lambda}, w^{\lambda}\right) \operatorname{sign}\left(u^{\lambda}-\bar{u}\right) \leq C .
$$

In view of the special expression of $G(\cdot, \cdot)$, we remark that

$$
G\left(u^{\lambda}, w^{\lambda}\right) \operatorname{sign}\left(u^{\lambda}-\bar{u}\right)=\left|G\left(u^{\lambda}, w^{\lambda}\right)\right|,
$$

so that finally

$$
\int_{\Omega}\left|u^{\lambda}-\bar{u}\right|(t)+\lambda \int_{Q_{t}}\left|G\left(u^{\lambda}, w^{\lambda}\right)\right| \leq C
$$

holds. Moreover in view of (1.2) we deduce that

$$
\iint_{Q_{T}}\left|w_{t}^{\lambda}\right|=\lambda \iint_{Q_{T}}\left|G\left(u^{\lambda}, w^{\lambda}\right)\right| \leq C_{1}
$$

which coincides with (3.20). Next we prove (3.21). Multiplying (1.1) by $u^{\lambda}-\bar{u}$ and integrating the result on $Q_{T}$ we obtain

$$
\iint_{Q_{T}} u_{t}^{\lambda}\left(u^{\lambda}-\bar{u}\right)=-\iint_{Q_{T}}\left|\nabla\left(u^{\lambda}-\bar{u}\right)\right|^{2}-\lambda \iint_{Q_{T}} G\left(u^{\lambda}, w^{\lambda}\right)\left(u^{\lambda}-\bar{u}\right)
$$

which implies that

$$
\iint_{Q_{T}}\left|\nabla\left(u^{\lambda}-\bar{u}\right)\right|^{2}=-\frac{1}{2} \iint_{Q_{T}} \frac{\partial}{\partial t}\left|u^{\lambda}-\bar{u}\right|^{2}-\lambda \iint_{Q_{T}} G\left(u^{\lambda}, w^{\lambda}\right)\left(u^{\lambda}-\bar{u}\right) \leq \lambda \iint_{Q_{T}}\left|G\left(u^{\lambda}, w^{\lambda}\right)\left(u^{\lambda}-\bar{u}\right)\right| .
$$

Using (3.20) and the fact that $u^{\lambda}$ is bounded we deduce (3.21).

Next we prove estimates of differences of space and time translates of $\left\{u^{\lambda}\right\}$. We set for $r \in \mathbf{R}^{+}$:

$$
\Omega_{r}=\{x \in \Omega, \quad B(x, 2 r) \subset \Omega\} .
$$

Lemma 3.3 There exists $C_{2}>0$ only depending on $T, \Omega$ and $\bar{u}$ such that

$$
\int_{0}^{T} \int_{\Omega_{r}}\left|u^{\lambda}(x+\xi, t)-u^{\lambda}(x, t)\right|^{2} d x d t \leq C_{2}|\xi|^{2},
$$

and

$$
\int_{0}^{T-\tau} \int_{\Omega}\left|u^{\lambda}(x, t+\tau)-u^{\lambda}(x, t)\right|^{2} d x d t \leq C_{2} \tau
$$

for all $\xi \in \mathbf{R}^{N},|\xi| \leq 2 r$ and $\tau \in(0, T)$. 
Proof. We first prove (3.22). We have that

$$
\begin{aligned}
\int_{0}^{T} \int_{\Omega_{r}}\left|u^{\lambda}(x+\xi, t)-u^{\lambda}(x, t)\right|^{2} d x d t & =\int_{0}^{T} \int_{\Omega_{r}}\left|\int_{0}^{1} \nabla u^{\lambda}(x+\sigma \xi, t) \cdot \xi d \sigma\right|^{2} d x d t \\
& \leq \int_{0}^{T} \int_{\Omega_{r}}\left[\int_{0}^{1}\left|\nabla u^{\lambda}(x+\sigma \xi, t)\right|^{2} d \sigma \int_{0}^{1}|\xi|^{2} d \sigma\right] d x d t \\
& \leq|\xi|^{2} \int_{0}^{1}\left[\int_{0}^{T}\left(\int_{\Omega_{r}}\left|\nabla u^{\lambda}(x+\sigma \xi, t)\right|^{2} d x\right) d t\right] d \sigma \\
& \leq|\xi|^{2} \int_{0}^{1}\left[\int_{0}^{T}\left(\int_{\Omega}\left|\nabla u^{\lambda}(y, t)\right|^{2} d y\right) d t\right] d \sigma \\
& \leq|\xi|^{2} \int_{0}^{T} \int_{\Omega}\left|\nabla u^{\lambda}(y, t)\right|^{2} d y d t
\end{aligned}
$$

Using (3.21) we deduce (3.22). Next we prove (3.23).

$$
\begin{aligned}
& \int_{0}^{T-\tau} \int_{\Omega}\left|u^{\lambda}(x, t+\tau)-u^{\lambda}(x, t)\right|^{2} d x d t \\
= & \int_{0}^{T-\tau} \int_{\Omega}\left(u^{\lambda}(x, t+\tau)-u^{\lambda}(x, t)\right)\left(\int_{0}^{\tau} \partial_{t} u^{\lambda}(x, t+\sigma) d \sigma\right) d x d t \\
= & \int_{0}^{T-\tau} \int_{\Omega}\left(u^{\lambda}(x, t+\tau)-u^{\lambda}(x, t)\right)\left(\int_{0}^{\tau}\left(\Delta u^{\lambda}-\lambda G\left(u^{\lambda}, w^{\lambda}\right)\right)(x, t+\sigma) d \sigma\right) d x d t \\
= & \int_{0}^{T-\tau} \int_{\Omega}\left(u^{\lambda}(x, t+\tau)-u^{\lambda}(x, t)\right)\left(\int_{0}^{\tau} \Delta u^{\lambda}(x, t+\sigma) d \sigma\right) d x d t \\
& -\lambda \int_{0}^{T-\tau} \int_{\Omega}\left(u^{\lambda}(x, t+\tau)-u^{\lambda}(x, t)\right)\left(\int_{0}^{\tau} G\left(u^{\lambda}, w^{\lambda}\right)(x, t+\sigma) d \sigma\right) d x d t \\
= & : I+I I
\end{aligned}
$$

Using (3.21) we have that

$$
\begin{aligned}
|I| & \leq \int_{0}^{\tau}\left(\int_{0}^{T-\tau} \int_{\Omega}\left|\nabla u^{\lambda}(x, t+\tau)-\nabla u^{\lambda}(x, t)\right|\left|\nabla u^{\lambda}(x, t+\sigma)\right| d x d t\right) d \sigma \\
& \leq 2 \tau \int_{0}^{T} \int_{\Omega}\left|\nabla u^{\lambda}(x, t)\right|^{2} d x d t \leq 2 \tau C_{1} .
\end{aligned}
$$

In view of (3.20) and Corollary 2.3 we obtain that

$$
|I I| \leq 2 K C_{1} \tau
$$

Finally substituting (3.25) and (3.26) into (3.24) we deduce (3.23).

Next we prove estimates of differences of space and time translates of $\left\{w^{\lambda}\right\}$. 
Lemma 3.4 There exists a positive function $h$ such that $h(\xi) \rightarrow 0$ as $\xi \rightarrow 0$ and:

$$
\int_{0}^{T} \int_{\Omega_{r}}\left|w^{\lambda}(x+\xi, t)-w^{\lambda}(x, t)\right| d x d t \leq h(\xi)
$$

and

$$
\int_{0}^{T-\tau} \int_{\Omega}\left|w^{\lambda}(x, t+\tau)-w^{\lambda}(x, t)\right| d x d t \leq C_{1} \tau
$$

for all $\xi \in \mathbf{R}^{N},|\xi| \leq 2 r$ and $\tau \in(0, T)$.

Proof. We first show (3.28). We have in view of the ordinary differential equation (1.2) for $w^{\lambda}$, that

$$
\begin{aligned}
\int_{0}^{T-\tau} \int_{\Omega}\left|w^{\lambda}(x, t+\tau)-w^{\lambda}(x, t)\right| d x d t & =\int_{0}^{T-\tau} \int_{\Omega}\left|\int_{0}^{\tau} \partial_{t} w^{\lambda}(x, t+\sigma) d \sigma\right| d x d t \\
& \leq \int_{0}^{\tau}\left(\int_{0}^{T} \int_{\Omega} \lambda\left|G\left(u^{\lambda}, w^{\lambda}\right)(x, t)\right| d x d t\right) d \sigma
\end{aligned}
$$

which with (3.20) gives (3.28). Next we prove (3.27). We first introduce the set

$$
\Omega_{r}^{\prime}=\cup_{x \in \Omega_{r}} B(x, r)
$$

and remark that by definition

$$
\Omega_{r} \subset \Omega_{r}^{\prime} \subset \Omega \text {. }
$$

As it is done in [6], we introduce the function $\psi \in C_{0}^{\infty}\left(\Omega_{r}^{\prime}\right)$, such that

$$
\psi(x)=\frac{4}{r} \int_{\Omega_{1}} \rho\left(\frac{4(y-x)}{r}\right) d y \quad \text { for all } x \in \Omega_{r}^{\prime},
$$

where $\Omega_{1}=\cup_{x \in \Omega_{r}} B(x, r / 4)$ and $\rho$ is the function defined by

$$
\rho(x)= \begin{cases}\rho_{0} \exp \left(-\frac{1}{1-|x|^{2}}\right) & \text { if }|x|<1, \\ 0 & \text { otherwise }\end{cases}
$$

and $\rho_{0}$ is a constant chosen such that $\int_{\mathbf{R}}^{N} \rho(x) d x=1$. One can check that $\psi$ satisfies that

$$
0 \leq \psi \leq 1 \text { in } \Omega_{r}^{\prime} \text { and } \psi=1 \text { in } \Omega_{r}
$$

Let $\xi \in \mathbf{R}^{N}$ with $|\xi| \leq r$. For all $(x, t) \in \Omega_{r}^{\prime} \times(0, T)$ we set

$$
\tilde{u}^{\lambda}(x, t)=u^{\lambda}(x+\xi, t) \text { and } \tilde{w}^{\lambda}(x, t)=w^{\lambda}(x+\xi, t) .
$$

Next we show that 


$$
E:=\left(G\left(u^{\lambda}, w^{\lambda}\right)-G\left(\tilde{u}^{\lambda}, \tilde{w}^{\lambda}\right)\right)\left(\operatorname{sign}\left(u^{\lambda}-\tilde{u}^{\lambda}\right)-\operatorname{sign}\left(w^{\lambda}-\tilde{w}^{\lambda}\right)\right) \geq 0, \text { a.e. in } \Omega_{r}^{\prime} \times(0, T) .
$$

We consider 4 different cases :

If $u^{\lambda}>\tilde{u}^{\lambda}$ and $w^{\lambda}>\tilde{w}^{\lambda}$ or if $u^{\lambda}<\tilde{u}^{\lambda}$ and $w^{\lambda}<\tilde{w}^{\lambda}$ then $\left(\operatorname{sign}\left(u^{\lambda}-\tilde{u}^{\lambda}\right)-\operatorname{sign}\left(w^{\lambda}-\tilde{w}^{\lambda}\right)\right)=0$ and thus $E=0$.

If $u^{\lambda} \geq \tilde{u}^{\lambda}$ and $w^{\lambda} \leq \tilde{w}^{\lambda}$ since $u \mapsto G(u, v)$ is increasing and $v \mapsto G(u, v)$ is non increasing we have that

$$
G\left(\tilde{u}^{\lambda}, \tilde{w}^{\lambda}\right) \leq G\left(u^{\lambda}, \tilde{w}^{\lambda}\right) \leq G\left(u^{\lambda}, w^{\lambda}\right)
$$

Thus $E \geq 0$.

Similarly if $u^{\lambda} \leq \tilde{u}^{\lambda}$ and $w^{\lambda} \geq \tilde{w}^{\lambda}$ then $E \geq 0$. This concludes the proof of (3.30). In view of the ordinary differential equation for $w^{\lambda},(1.2)$, we deduce from (3.30) that

$$
\left(w^{\lambda}-\tilde{w}^{\lambda}\right)_{t}\left(\operatorname{sign}\left(u^{\lambda}-\tilde{u}^{\lambda}\right)-\operatorname{sign}\left(w^{\lambda}-\tilde{w}^{\lambda}\right)\right) \geq 0, \text { a.e. in } \Omega_{r}^{\prime} \times(0, T) .
$$

Multiplying the equality

$$
\left(u^{\lambda}-\tilde{u}^{\lambda}\right)_{t}+\left(w^{\lambda}-\tilde{w}^{\lambda}\right)_{t}-\Delta\left(u^{\lambda}-\tilde{u}^{\lambda}\right)=0
$$

by $\left[\operatorname{sign}^{\delta}\left(u^{\lambda}-\tilde{u}^{\lambda}\right)\right] \psi$ and integrating by part on $\Omega_{r}^{\prime} \times(0, t)$ for $t \in(0, T)$ we obtain that

$$
\begin{array}{r}
\int_{0}^{t} \int_{\Omega_{r}^{\prime}}\left(u^{\lambda}-\tilde{u}^{\lambda}\right)_{t}\left[\operatorname{sign}^{\delta}\left(u^{\lambda}-\tilde{u}^{\lambda}\right)\right] \psi d x d s+\int_{0}^{t} \int_{\Omega_{r}^{\prime}}\left(w^{\lambda}-\tilde{w}^{\lambda}\right)_{t}\left[\operatorname{sign}^{\delta}\left(u^{\lambda}-\tilde{u}^{\lambda}\right)\right] \psi d x d s \\
+\int_{0}^{t} \int_{\Omega_{r}^{\prime}} \nabla\left(u^{\lambda}-\tilde{u}^{\lambda}\right) \nabla\left\{\left[\operatorname{sign}^{\delta}\left(u^{\lambda}-\tilde{u}^{\lambda}\right)\right] \psi\right\} d x d s=0
\end{array}
$$

which by Lebesgue's dominated convergence theorem implies that

$$
\begin{aligned}
\int_{0}^{t} \int_{\Omega_{r}^{\prime}}\left(u^{\lambda}-\tilde{u}^{\lambda}\right)_{t}\left[\operatorname{sign}\left(u^{\lambda}-\tilde{u}^{\lambda}\right)\right] & \psi d x d s+\int_{0}^{t} \int_{\Omega_{r}^{\prime}}\left(w^{\lambda}-\tilde{w}^{\lambda}\right)_{t}\left[\operatorname{sign}\left(u^{\lambda}-\tilde{u}^{\lambda}\right)\right] \psi d x d s \\
& +\int_{0}^{t} \int_{\Omega_{r}^{\prime}} \nabla\left(u^{\lambda}-\tilde{u}^{\lambda}\right) \operatorname{sign}\left(u^{\lambda}-\tilde{u}^{\lambda}\right) \nabla \psi d x d s \leq 0 .
\end{aligned}
$$

This with (3.31) gives that

$$
\int_{0}^{t} \int_{\Omega_{r}^{\prime}}\left|u^{\lambda}-\tilde{u}^{\lambda}\right|_{t} \psi d x d s+\int_{0}^{t} \int_{\Omega_{r}^{\prime}}\left|w^{\lambda}-\tilde{w}^{\lambda}\right|_{t} \psi d x d s+\int_{0}^{t} \int_{\Omega_{r}^{\prime}} \nabla\left|u^{\lambda}-\tilde{u}^{\lambda}\right| \nabla \psi d x d s \leq 0
$$


which implies after integration in time in the two first terms that

$$
\begin{aligned}
& \int_{\Omega_{r}^{\prime}}\left(\left|\left(u^{\lambda}-\tilde{u}^{\lambda}\right)(x, t)\right|+\left|\left(w^{\lambda}-\tilde{w}^{\lambda}\right)(x, t)\right|\right) \psi(x) d x \\
& \leq \int_{\Omega_{r}^{\prime}}\left(\left|u_{0}(x)-u_{0}(x+\xi)\right|+\left|w_{0}(x)-w_{0}(x+\xi)\right|\right) \psi(x) d x+\int_{0}^{t} \int_{\Omega_{r}^{\prime}}\left|\left(u^{\lambda}-\tilde{u}^{\lambda}\right)(x, t)\right||\Delta \psi(x)| d x d s
\end{aligned}
$$

Integrating this inequality with respect to $t$ on $(0, T)$ and using the fact that $0 \leq \psi \leq 1$ in $\Omega_{r}^{\prime}$ and $\psi=1$ in $\Omega_{r}$ we deduce that

$$
\begin{aligned}
& \int_{0}^{T} \int_{\Omega_{r}}\left(\left|\left(u^{\lambda}-\tilde{u}^{\lambda}\right)(x, t)\right|+\left|\left(w^{\lambda}-\tilde{w}^{\lambda}\right)(x, t)\right|\right) d x d t \\
& \leq T \int_{\Omega_{r}^{\prime}}\left(\left|u_{0}(x)-u_{0}(x+\xi)\right|+\left|w_{0}(x)-w_{0}(x+\xi)\right|\right) d x \\
& +T\left(\int_{0}^{T} \int_{\Omega_{r}^{\prime}}\left|u^{\lambda}-\tilde{u}^{\lambda}\right|^{2}\right)^{1 / 2}\left(\int_{0}^{T} \int_{\Omega_{r}^{\prime}}|\Delta \psi|^{2}\right)^{1 / 2} .
\end{aligned}
$$

Also using (3.22) with $\Omega_{r}$ replaced by $\Omega_{r}^{\prime}$ we deduce that

$$
\begin{aligned}
\int_{0}^{T} \int_{\Omega_{r}}\left|\left(w^{\lambda}-\tilde{w}^{\lambda}\right)(x, t)\right| d x d t \leq & T \int_{\Omega_{r}^{\prime}}\left(\left|u_{0}(x)-u_{0}(x+\xi)\right|+\left|w_{0}(x)-w_{0}(x+\xi)\right|\right) d x \\
& +C(T)|\xi|\left(\int_{\Omega_{r}^{\prime}}|\Delta \psi|^{2}\right)^{1 / 2}
\end{aligned}
$$

Therefore we have proved (3.27) with $h(\xi)$ being equal to the right hand-side of (3.33).

Corollary 3.5 Let $\left(u^{\lambda}, w^{\lambda}\right)$ be the unique nonnegative solution of Problem $\left(P^{\lambda}\right)$. There exist subsequences $\left\{u^{\lambda_{m}}\right\}$ and $\left\{w^{\lambda_{m}}\right\}$ and functions $U \in L^{\infty}\left(Q_{T}\right)$ and $W \in L^{\infty}\left(Q_{T}\right)$ such that

$$
u^{\lambda_{m}} \rightarrow U \text { and } w^{\lambda_{m}} \rightarrow W
$$

strongly in $L^{2}\left(Q_{T}\right)$ as $\lambda_{m}$ tends to $\infty$. Moreover as $\lambda_{m}$ tends to $\infty, u^{\lambda_{m}} \rightarrow U$ weakly in $L^{2}\left(0, T ; H^{1}(\Omega)\right)$.

Proof. The first part of Corollary 3.5 follows from Lemmas 3.3 and 3.4 and the Riesz-FréchetKolmogorov theorem ([4] Theorem IV.25 and Corollary IV.26). The last assertion follows from (3.21). 
Lemma 3.6 We have that $G(U, W)=0$ so that setting $Z:=-(U+W)+\bar{u}$ we obtain

$$
\left\{\begin{array}{l}
\text { if } U \geq \bar{u} \text { then } U=\bar{u} \text { and } Z=-W \\
\text { if } U<\bar{u} \text { then } W=0 \text { and } Z=-U+\bar{u}
\end{array}\right.
$$

Thus $Z^{+}=-U+\bar{u}$.

Proof. We set

$$
G_{\varepsilon}(u, w):=(u-\bar{u})^{+}-\operatorname{sign}_{\varepsilon}^{+}(w)(u-\bar{u})^{-},
$$

where $\operatorname{sign}_{\varepsilon}^{+}$is the continuous function defined by

$$
\operatorname{sign}_{\varepsilon}^{+}(x)= \begin{cases}1 & \text { if } x \geq \varepsilon \\ \frac{1}{\varepsilon} x & \text { if } 0 \leq x \leq \varepsilon \\ 0 & \text { if } x \leq 0\end{cases}
$$

Next we check that

$$
0 \leq(u-\bar{u}) G_{\varepsilon}(u, w) \leq(u-\bar{u}) G(u, w),
$$

for all $(u, w) \in \mathbf{R}^{2}$. We consider three cases :

If $w \geq \varepsilon$ then $G(u, w)=G_{\varepsilon}(u, w)=u-\bar{u}$ so that

$$
0 \leq(u-\bar{u}) G_{\varepsilon}(u, w) \leq(u-\bar{u}) G(u, w) .
$$

If $0 \leq w \leq \varepsilon$ and $u-\bar{u} \geq 0$ then $(u-\bar{u}) G_{\varepsilon}(u, w)=(u-\bar{u})^{2}=(u-\bar{u}) G(u, w) \geq 0$.

If $0 \leq w \leq \varepsilon$ and $u-\bar{u} \leq 0$ then we have $(u-\bar{u}) G_{\varepsilon}(u, w)=\frac{1}{\varepsilon} w(u-\bar{u})^{2} \geq 0$ and moreover

$$
(u-\bar{u}) G_{\varepsilon}(u, w) \leq(u-\bar{u}) G(u, w) .
$$

This concludes the proof of (3.34). Applying (3.34) at the point $\left(u^{\lambda_{m}}, w^{\lambda_{m}}\right)$ and integrating the result on $Q_{T}$ we deduce that

$$
0 \leq \int_{Q_{T}}\left(u^{\lambda_{m}}-\bar{u}\right) G_{\varepsilon}\left(u^{\lambda_{m}}, w^{\lambda_{m}}\right) \leq \int_{Q_{T}}\left(u^{\lambda_{m}}-\bar{u}\right) G\left(u^{\lambda_{m}}, w^{\lambda_{m}}\right)
$$

In view of Corollary 3.5 and (3.20) we deduce that $\lim _{\lambda_{m} \uparrow \infty} \int_{Q_{T}}\left(u^{\lambda_{m}}-\bar{u}\right) G\left(u^{\lambda_{m}}, w^{\lambda_{m}}\right)=0$ and thus since $G_{\varepsilon}$ is continuous we have that

$$
\int_{Q_{T}}(U-\bar{u}) G_{\varepsilon}(U, W)=0
$$

Therefore $G_{\varepsilon}(U, W)=0$ or $U=\bar{u}$. Finally letting $\varepsilon$ tend to 0 we obtain that $G(U, W)=0$ or $U=\bar{u}$, which concludes the proof of Lemma 3.6.

Lemma 3.7 The function $Z:=-(U+W)+\bar{u}$ is a weak solution of Problem $(S P)$. 
Proof. Let $\left(u^{\lambda_{m}}, w^{\lambda_{m}}\right)$ be the unique solution of Problem $\left(P^{\lambda_{m}}\right)$. Then

$$
\int_{Q_{T}}\left(u^{\lambda_{m}}+w^{\lambda_{m}}\right) \xi_{t}-\int_{Q_{T}} \nabla u^{\lambda_{m}} \nabla \xi=-\int_{\Omega}\left(u_{0}+w_{0}\right) \xi(0),
$$

for all $\xi \in C^{2,1}\left(\overline{Q_{T}}\right)$ such that $\xi(T)=0$. Letting $\lambda_{m}$ tends to $\infty$ we deduce that

$$
\int_{Q_{T}}(U+W) \xi_{t}-\int_{Q_{T}} \nabla U \cdot \nabla \xi=-\int_{\Omega}\left(u_{0}+w_{0}\right) \xi(0)
$$

for all $\xi \in C^{2,1}\left(\overline{Q_{T}}\right)$ such that $\xi(T)=0$.

We consider now the function $Z=-(U+W)+\bar{u}$, then by Lemma 3.6 we deduce that $Z$ is a weak solution of Problem $(S P)$ in the following sense : (i) $Z \in L^{\infty}\left(Q_{T}\right)$; (ii) $Z$ satisfies the following equality

$$
\int_{Q_{T}} Z \xi_{t}-\int_{Q_{T}} \nabla Z^{+} \cdot \nabla \xi=\int_{\Omega}\left(u_{0}+w_{0}-\bar{u}\right) \xi(0)
$$

Lemma 3.8 The function $Z:=-(U+W)+\bar{u}$ is the weak solution of Problem $(S P)$ and the whole sequence $\left(u^{\lambda}, w^{\lambda}\right)$ converges to $(U, W)=\left(-Z^{+}+\bar{u}, Z^{-}\right)$as $\lambda$ tends to $\infty$.

Proof. Lemma 3.8 follows directly from the uniqueness of the weak solution of Problem $(S P)$ (see [9] Proposition 5) and the fact that the functions $U=-Z^{+}+\bar{u}, W=Z^{-}$are uniquely defined as well. 


\section{References}

[1] N.D. Alikakos, P. Hess, H. Matano: Discrete order preserving semigroups and stability for periodic parabolic differential equations. J. Differ. Equations 82, 322-341 (1989).

[2] D. Bothe and D. Hilhorst, A reaction-diffusion system with fast reversible reaction, J. Math. Anal. Appl., 286, (2003), 125-135.

[3] N. Bouillard, R. Eymard, R. Herbin and P. Montarnal, Diffusion with dissolution and precipitation in a porous media: Mathematical analysis and numerical approximation of a simplified model, Mathematical Modelling and Numerical Analysis, submitted.

[4] H. Brezis, Analyse fonctionnelle, Masson, Paris, 1983.

[5] M. Crandall and M. Pierre, Regularizing Effect for $u_{t}+E \phi(u)=0$ in $L^{1}$, Journal of Functional Analysis 45 (1982), 194-212.

[6] R. Eymard, D. Hilhorst, R. van der Hout and L.A. Peletier, A reaction-diffusion system approximation of a one-phase Stefan problem, Optimal control and Partial Differential Equations, IOS Press (2001).

[7] R. Eymard, T. Gallouët, R. Herbin, Finite Volume Method, Handbook for Numerical Analysis, P.G Ciarlet, J.L. Lions Eds, North Holland, 7, (2000), 715-1022.

[8] R. Eymard, T. Gallouët, R. Herbin, D. Hilhorst and M. Mainguy, Intantaneous and noninstantaneous dissolution : approximation by the finite volume method, ESAIM Proceedings, (1998).

[9] D. Hilhorst, J.R. King and M. Röger, Mathematical Analysis of a model describing the invasion of bacteria in burn wounds, to appear in Nonlinear Analysis TMA.

[10] J. Pousin, Infinitely fast kinetics for dissolution and diffusion in open reactive systems, Nonlinear analysis 39 (2000), 261-279. 